\title{
TOPOLOGICAL CONJUGACY OF AFFINE TRANSFORMATIONS OF TORI
}

\author{
BY \\ PETER WALTERS
}

0. Introduction. Let $K^{n}$ denote the $n$-dimensional torus. Let $T$ be an affine transformation of $K^{n}$ onto $K^{n}$ and $S$ an affine transformation of $K^{m}$ onto $K^{m}$ for which there exists a continuous map $h$ of $K^{n}$ onto $K^{m}$ satisfying $h T=S h$. R. L. Adler and R. Palais have proved that, in the special cases when $m=n$ and $T$ and $S$ are ergodic automorphisms of $K^{n}$, all homeomorphism $g$ of $K^{n}$, satisfying $g T=S g$, are affine transformations [1]. The main purpose of this paper is to obtain necessary and sufficient conditions for the existence of a nonaffine continuous map $g$ of $K^{n}$ onto $K^{m}$ such that $g T=S g$. (Theorem 2, §4). The conditions obtained are unchanged under the requirements that $m=n$ and $T, S, h$ and $g$ be invertible transformations of $K^{n}$.

We obtain the above-mentioned result of Adler and Palais as a corollary of Theorem 2. We also prove that two automorphisms $T$ and $S$ of $K^{n}$ must be ergodic if all homeomorphisms $g$ of $K^{n}$ such that $g T=S g$ are affine transformations. (We require, of course, that there exist homeomorphisms $g$ such that $g T=S g$.) Another corollary of Theorem 2 concerns affine transformations with quasi-discrete spectrum. A. H. M. Hoare and W. Parry have shown that every minimal affine transformation of a compact, connected, metric, abelian group $X$ has quasidiscrete spectrum, and that if $T$ and $S$ are minimal affine transformations of $X$ then all homeomorphisms $g$ of $X$, such that $g T=S g$, are affine [5], [6]. Our corollary is restricted to toroidal groups but extends Hoare and Parry's result in this case.

Theorem 2 is also used to obtain a necessary and sufficient condition for a strong-mixing affine transformation of $K^{n}$ to have a continuous $p$ th root (Theorem $3, \S 5)$. In Theorem 1 a necessary and sufficient condition for an affine transformation of a compact, connected, metric, abelian group to be ergodic is given.

I should like to express my gratitude to Dr. W. Parry for supervising this work and also to Dr. D. E. Cohen and Dr. A. H. M. Hoare for supplying useful examples. My thanks are also due to the referee for some helpful suggestions.

1. Preliminaries. If $X$ is a compact, connected, metric, abelian group (with additive notation) then $\hat{X}$ will denote its discrete, torsion free, countable character group (with multiplicative notation) [7].

Let $X$ and $Y$ be compact, connected, metric, abelian groups. A homomorphism of $X$ onto $Y$ is a continuous mapping of $X$ onto $Y$ which is an abstract group

Received by the editors September 9, 1966 and, in revised form, December 9, 1966. 
homomorphism. A homomorphism of $X$ onto itself is called an endomorphism of $X$, and a one-to-one endomorphism is called an automorphism. An affine transformation of $X$ onto $Y$ is a transformation of the form $T(x)=a+A(x), x \in X$, where $a \in Y$ and $A$ is a homomorphism of $X$ onto $Y$. Clearly every homomorphism is an affine transformation. An affine transformation is a continuous, Haar measure-preserving transformation.

An endomorphism $A$ of $X$ onto $X$ induces a one-to-one dual endomorphsim, which we also denote by $A$, of $\hat{X}$ into $\hat{X}$ defined by $(A \gamma)(x)=\gamma(A x), \gamma \in \hat{X}, x \in X$. A. H. M. Hoare and W. Parry have shown that an affine transformation $T=a+A$ of $X$ onto $X$ is ergodic if and only if $\gamma \in \hat{X}, A^{n} \gamma=\gamma$, for some $n>0 \Rightarrow A \gamma=\gamma$, and $[a,(A-I) X]=X$, where $[a,(A-I) X]$ denotes the smallest closed subgroup of $X$ containing $a$ and $(A-I) X$ [4]. (See also [2].) For the special case of an endomorphism (i.e. $a=0$ ) these conditions reduce to the well-known condition of $\mathrm{P}$. R. Halmos, namely, $A$ is ergodic if and only if $\gamma \in \hat{X}, A^{n} \gamma=\gamma$, for some $n>0 \Rightarrow \gamma \equiv 1$ [3]. Halmos' condition is also true when $X$ is not connected. If $T=a+A$ is ergodic then $T^{n}$ is ergodic for every positive integer $n$ i.e. $T$ is totally ergodic. It can be shown easily from the above ergodicity conditions that $T=a+A$ is strong-mixing if and only if $A$ is ergodic. F. J. Hahn has proved this result for groups which are not necessarily connected [2]. In particular we have another well-known result of Halmos, that an endomorphism of $X$ is strong-mixing if and only if it is ergodic.

The Hilbert space consisting of all measurable square-integrable complexvalued functions of $X$, will be denoted by $L^{2}(X)$. The elements of $\hat{X}$ form a basis for $L^{2}(X) . R^{n}$ will denote $n$-dimensional real Euclidean space and $I$ the identity operator on $R^{n}$, i.e. $I(x)=x$ for all $x \in R^{n}$. $R^{n}$ will be thought of as a subset of $n$-dimensional complex space $C^{n}$. If $L$ is a linear transformation of $R^{n}$ onto $R^{m}$ we can define a linear transformation, which we also denote by $L$, of $C^{n}$ onto $C^{m}$ by $L(x+i y)=L x+i L y, x, y \in R^{n}$. If $P(x)=\left(p_{1}(x), \ldots, p_{m}(x)\right)$ is a transformation of $R^{n}$ into $R^{m}$ then $k P(x), k \in R$ will denote the transformation defined by $k P(x)$ $=\left(k p_{1}(x), \ldots, k p_{m}(x)\right) . \mathscr{R}(\mu)$ and $\mathscr{I}(\mu)$ will denote the real and imaginary parts of the complex number $\mu$ and if $P(x)=\left(p_{1}(x), \ldots, p_{m}(x)\right)$ is a transformation of $R^{n}$ into $C^{m}$ then $\mathscr{R} P(x)$ and $\mathscr{I} P(x)$ will denote the transformations of $R^{n}$ into $R^{m}$ defined by $\mathscr{R} P(x)=\left(\mathscr{R} p_{1}(x), \ldots, \mathscr{R} p_{m}(x)\right)$ and $\mathscr{I} P(x)=\left(\mathscr{I} p_{1}(x), \ldots, \mathscr{I} p_{m}(x)\right)$ respectively.

2. The lifting procedure. In this section we describe a method of "lifting" a continuous map of $K^{n}$ into $K^{m}$ to a continuous map of $R^{n}$ into $R^{m}$ which can be uniquely written as the sum of a constant map, a linear map, and a map which is periodic over the subset $Z^{n}$ of $R^{n}$ consisting of points with integer coordinates. This technique is employed in the proof of Theorem 2. The account given here is modelled on that in [1].

$R^{n}$ is the universal covering group of $K^{n}\left[7\right.$, p. 232]. We have $K^{n}=R^{n} / Z^{n}$. Let $\pi_{n}: R^{n} \rightarrow K^{n}$ be the universal covering map defined by $\pi_{n}(x)=x+Z^{n} . \pi_{n}$ is an open, continuous homomorphism of $R^{n}$ onto $K^{n}$ [7].

Suppose $g: K^{n} \rightarrow K^{m}$ is a continuous map. There is a unique point $c=\left(c_{1}, \ldots, c_{m}\right)$ 
$\in R^{m}$ with $0 \leqq c_{i}<1, i=1,2, \ldots, m$, such that $\pi_{m}(c)=g(0)$ and a unique continuous map $F: R^{n} \rightarrow R^{m}$ with $F(0)=c$ such that $\pi_{m} F=g \pi_{n}$ (i.e. $F$ is obtained by lifting $g$ ). If $H(x)=F(x)-c, x \in R^{n}$, then $H Z^{n} \subset Z^{m}$ and $\left.H\right|_{z^{n}}$, being a homomorphism of $Z^{n}$ into $Z^{m}$, extends uniquely to a linear map $L$ of $R^{n}$ into $R^{m}$. Put $P(x)=F(x)$ $-L(x)-c, x \in R^{n}$. Then $P(0)=0$ and $P(x+\nu)=P(x), x \in R^{n}, \nu \in Z^{n}$. The last relation holds because the continuous map $P(x+\nu)-P(x)$, for a fixed $\nu \in Z^{n}$, maps the connected space $R^{n}$ into the discrete set $Z^{m}$.

Therefore to every continuous map $g: K^{n} \rightarrow K^{m}$ we have assigned a continuous map $F: R^{n} \rightarrow R^{m}$ and $F(x)=L(x)+P(x)+c$, where $c=\left(c_{1}, \ldots, c_{m}\right) \in R^{m} 0 \leqq c_{i}<1$, $i=1, \ldots, m, L$ is linear, $L Z^{n} \subset Z^{m}$, and $P$ is a continuous map satisfying $P(0)=0$ and $P(x+\nu)=P(x), x \in R^{n}, \nu \in Z^{n}$. Moreover, this decomposition is easily seen to be unique. $L$ is called the linear part, $P$ the periodic part and $c$ the constant part of the lifting $F$, and we write $F_{g}(x)=L_{g}(x)+P_{g}(x)+c_{g}$ to show that $F_{g}$ is the lifting of $g$.

If $g: K^{n} \rightarrow K^{m}$ and $h: K^{m} \rightarrow K^{r}$ are continuous, we have $F_{h g}(x)=F_{h} F_{g}(x)+\nu$, $x \in R^{n}$, where $\nu$ is some fixed element of $Z^{r}$. By the uniqueness of the decomposition of a lifting we have

(1) $L_{h g}(x)=L_{h} L_{g}(x)$

(2) $P_{h g}(x)=L_{h} P_{g}(x)+P_{h}\left(L_{g}(x)+P_{g}(x)+c_{g}\right)-P_{h}\left(c_{g}\right)$,

(3) $c_{h g}=L_{h}\left(c_{g}\right)+P_{h}\left(c_{g}\right)+c_{h}+\nu$.

$A$ is a homomorphism of $K^{n}$ onto $K^{m}$ if and only if the lifting of $A$ is a linear transformation of $R^{n}$ onto $R^{m}$. In this case the lifting of $A$ will also be denoted by $A$. An endomorphism $A$ of $K^{n}$ onto $K^{n}$ is an automorphism if and only if the lifted transformation $A$ is an invertible linear transformation of $R^{n}$ such that $A Z^{n}=Z^{n}$. An endomorphism $A$ of $K^{n}$ is ergodic if and only if the lifted transformation $A$ has no roots of unity as eigenvalues. In fact if there exists $\gamma \in \hat{K}^{n}$, $\gamma \not \equiv 1$, such that $A^{p} \gamma=\gamma$ then the lifted transformation $A$ has a $p$ th root of unity as an eigenvalue and conversely [3, p. 55].

$T$ is an affine transformation of $K^{n}$ onto $K^{m}$ if and only if the lifting of $T$ is the sum of a constant transformation and a linear transformation of $R^{n}$ onto $R^{m}$. If $T=a+A$ then the lifting of $T$ will also be denoted by $a+A$. An affine transformation $T=a+A$ of $K^{n}$ onto $K^{n}$ is one-to-one if and only if the lifted transformation $A$ is an invertible linear transformation of $R^{n}$ such that $A Z^{n}=Z^{n}$. From the ergodicity conditions stated in $\S 1$ we see that the affine transformation $T=a+A$ of $K^{n}$ onto $K^{n}$ is ergodic if and only if $\left[a,(A-I) K^{n}\right]=K^{n}$, and the lifted transformation $A$ has no roots of unity as eigenvalues except possibly $\lambda=1$.

3. Ergodicity for affine transformations. It is well known that if a measure space $X$ is a topological space with a countable base, such that every nonempty open set has positive measure, and if $T$ is an ergodic measure-preserving transformation on $X$, then for almost every $x \in X$ the orbit of $x$ (i.e. the set $\left\{T^{n} x \mid n=0,1,2, \ldots\right\}$ ) is dense in $X$ [3, p. 26]. We show below that for affine transformations on compact, connected, metric, abelian, groups the existence of a dense orbit is also sufficient for ergodicity. 
THEOREM 1. If $X$ is a compact, connected, metric, abelian group and $T=a+A$ an affine transformation of $X$, then $T$ is ergodic if and only if there exists a point $x_{0} \in X$ such that the set $\left\{T^{n} x_{0} \mid n=1,2, \ldots\right\}$ is dense in $X$.

Proof. Suppose that $T$ is ergodic. Let $\left\{U_{n}\right\}$ be a countable basis for the open sets of $X$. The set of points $E_{i}$ whose orbits do not meet a particular $U_{i}$ is measurable, and since $m\left(U_{i}\right)>0$ and $T$ is ergodic we have $m\left(E_{i}\right)=0$. Hence the complement of $\bigcup_{i=1}^{\infty} E_{i}$, the set of points with dense orbits, has measure one.

Conversely suppose that for some $x_{0} \in X$ the set $\left\{T^{n} x_{0} \mid n=0,1, \ldots\right\}$ is dense in $X$. We shall show that the ergodicity conditions stated in $\S 1$ are satisfied. If $[a,(A-I) X] \neq X$, there exists $\gamma \in \hat{X}, \gamma \neq \equiv 1$ such that $\gamma(a)=1$ and $\gamma(A-I) X=1$. Then $\gamma\left(T^{n} x_{0}\right)=\gamma\left(x_{0}\right)$ for all $n \geqq 0$ and hence $\gamma \equiv 1$; a contradiction.

Suppose $A^{m} \gamma=\gamma$ for some $m>1, \gamma \in \hat{X}$. If $\gamma_{1}(x)=\gamma(A-I)(x)$, then $\gamma_{1} \in \hat{X}$ and $\gamma_{1}\left(T^{m} x\right)=\gamma_{1}(x)$ for all $x \in X$. Hence $\gamma_{1}$ assumes only the values $\gamma_{1}\left(x_{0}\right), \gamma_{1}\left(T x_{0}\right), \ldots$, $\gamma_{1}\left(T^{m-1} x_{0}\right)$ on the dense set $\left\{T^{n} x_{0} \mid n=0,1, \ldots\right\}$. Therefore $\gamma_{1}$ assumes only these values on $X$ and since $X$ is connected we have $\gamma_{1} \equiv 1$, i.e. $A \gamma=\gamma$. This completes the proof.

COROLlary. A minimal affine transformation of a compact, connected, metric, abelian group is ergodic.

Proof. A homeomorphism of a compact Hausdorff space $X$ is defined to be minimal if for each point $x \in X$ the set $\left\{T^{n} x \mid n=0,1,2, \ldots\right\}$ is dense in $X$. The corollary follows from Theorem 1.

4. Topological conjugacy for affine transformations. In this section we prove Theorem 2, the main result of the paper. First we prove two lemmas that are needed for the proof of Theorem 2.

LeMma 1. If $L$ is a linear transformation of $R^{n}$ onto $R^{m}(m \leqq n)$ and $P$ is a continuous map of $R^{n}$ onto a compact subset of $R^{m}$ then $L+P$ maps $R^{n}$ onto $R^{m}$.

Proof. By considering the rank of $L$ and using the fact that $L$ maps $R^{n}$ onto $R^{m}$ we can reduce the proof to the case $m=n$ where $L$ is invertible. It suffices to show that $I+Q$ maps $R^{n}$ onto $R^{n}$ where $Q=L^{-1} P$ maps $R^{n}$ onto a compact subset of $R^{n}$. We have to show that for every $y \in R^{n}$ there exists $x \in R^{n}$ such that $x+Q(x)=y$, i.e. the compact map $y-Q(\cdot)$ has a fixed point and this follows from the Brouwer fixed point theorem by considering a closed sphere containing the range of $y-Q(\cdot)$.

Lemma 2. Let $L$ be an invertible linear transformation of $R^{n}$ and $P: R^{n} \rightarrow R^{n} a$ continuous transformation which satisfies a Lipschitz condition of the form

$$
\|P(x)-P(y)\| \leqq M\|x-y\|
$$

for $x, y \in R^{n}$, where $M$ is a constant and $\|\cdot\|$ denotes the ordinary euclidean norm. Then there exists a real number $\varepsilon>0$ such that $L+\varepsilon P$ maps $R^{n}$ in a one-to-one manner into itself.

Proof. It suffices to consider the case when $L=I$, the identity linear transformation of $R^{n}$. Choose $\varepsilon>0$ such that $\varepsilon M<1$. Then $x+\varepsilon P(x)=y+\varepsilon P(y)$ implies 
$\|x-y\|=\varepsilon\|P x-P y\| \leqq \varepsilon M\|x-y\|$ which implies that $x=y$. Hence $I+\varepsilon P$ is oneto-one.

In $\S 2$ we saw that the endomorphisms of $K^{n}$ onto $K^{n}$ are in one-to-one correspondence with the invertible linear transformations of $R^{n}$ which preserve $Z^{n}$. Therefore if a fixed basis is chosen in $K^{n}$ the endomorphisms of $K^{n}$ onto $K^{n}$ are in one-to-one correspondence with the invertible $n \times n$ matrices with integer entries. If $A$ is an endomorphism of $K^{n}$ let [A] denote the corresponding matrix. If $[A]=\left[a_{i j} \mid i, j=1,2, \ldots, n\right]$ then the endomorphism $A$ is given by

$$
A\left(\left(x_{1}, \ldots, x_{n}\right)+Z^{n}\right)=\left(\left(\sum_{j=1}^{n} a_{1 j} x_{j}, \sum_{j=1}^{n} a_{2 j} x_{j}, \ldots, \sum_{j=1}^{n} a_{n j} x_{j}\right)+Z^{n}\right) .
$$

The lifted transformation $A$ is given by

$$
A\left(x_{1}, \ldots, x_{n}\right)=\left(\sum_{j=1}^{n} a_{1 j} x_{j}, \sum_{j=1}^{n} a_{2 j} x_{j}, \ldots, \sum_{j=1}^{n} a_{n j} x_{j}\right), \quad\left(x_{1}, \ldots, x_{n}\right) \in R^{n} .
$$

Therefore the eigenvalues of the linear transformation $A$ of $R^{n}$ are the eigenvalues of the matrix $[A]$.

THEOREM 2. Let $T=a+A$ be an affine transformation of $K^{n}$ onto $K^{n}$ and $S=b+B$ an affine transformation of $K^{m}$ onto $K^{m}$. Suppose there exists a continuous map $h$ of $K^{n}$ onto $K^{m}$ such that $h T=S h$. Then there exists a nonaffine, continuous map $g$ of $K^{n}$ onto $K^{m}$ such that $g T=S g$ if and only if there exists $\gamma \in \hat{K}^{n}, \gamma \neq 1$, and an eigenvalue $\lambda$ of the lifted transformation $B$ such that $|\lambda|=1, A^{p} \gamma=\gamma$ for some integer $p \geqq 1$, and for all such $p, \gamma\left(a+A(a)+\cdots+A^{p-1}(a)\right)=\lambda^{p}$.

In the cases when $m=n$, and $T, S, h$ are invertible transformations the above conditions are necessary and sufficient for the existence of a nonaffine homeomorphism $g$ of $K^{n}$ such that $g T=S g$.

Proof. We first prove the necessity of the conditions. The idea of the proof is to lift the problem to euclidean spaces and use eigenvalue theory in $C^{m}$ to bring in an eigenvalue $\lambda$ of the lifted transformation $B$. We then produce a nonconstant, continuous, complex-valued function $q(z)$ of $K^{n}$ such that $q(T z)=\lambda q(z)$, and use the fact that the elements of $\hat{K}^{n}$ form a basis for $L^{2}\left(K^{n}\right)$ to obtain the required conditions.

Suppose $g$ is a nonaffine, continuous map of $K^{n}$ onto $K^{m}$ such that $g T=S g$. Let $F_{g}(x)=L(x)+P(x)+c, x \in R^{n}$, be the decomposition of the lifting of $g$ into linear, periodic and constant parts. Since $g$ is nonaffine, $P \not \equiv 0$ and by equation (2)

$$
P(a+A(x))=P(a)+B P(x), \quad x \in R^{n} .
$$

There exists an invertible linear transformation $U: C^{m} \rightarrow C^{m}$ such that $U^{-1} B U$ $=D_{B}$ is the Jordan normal form of the linear transformation $B$. If $Q(x)=U^{-1} P(x)$, $x \in R^{n}$, applying $U^{-1}$ to the above equation gives

$$
Q(a+A(x))=Q(a)+D_{B} Q(x) \quad x \in R^{n} .
$$

$Q: R^{n} \rightarrow C^{m}$ is continuous, $Q \not \equiv 0, Q(0)=0$ and $Q(x+\nu)=Q(x), x \in R^{n}, \nu \in Z^{n}$. 
Let $w_{1}, w_{2}, \ldots, w_{m}$ be the basis of $C^{m}$ for which the linear transformation $B$ has Jordan normal form $D_{B}$. Suppose $Q(x)=\sum_{i=1}^{m} f_{i}(x) w_{i}, x \in R^{n}$, where the functions $f_{i}$ are complex-valued. If $j_{0}$ is the smallest integer for which $f_{j_{0}} \not \equiv 0$, then

$$
f_{j_{0}}(a+A(x))=f_{j_{0}}(a)+\lambda f_{j_{0}}(x) \quad x \in R^{n},
$$

where $\lambda$ is the eigenvalue of the linear transformation $B$ corresponding to the basis element $w_{j_{0}}$. Since $f_{j_{0}}(x+\nu)=f_{j_{0}}(x), x \in R^{n}, \nu \in Z^{n}, f_{j_{0}}$ determines a continuous map $l: K^{n} \rightarrow C$ such that $l \not \equiv 0, l(0)=0$ and $l(T z)=l(a)+\lambda l(z), z \in K^{n}$. Hence $\int l(z) d m=l(a)+\lambda \int l(z) d m$, where $m$ denotes normalized Haar measure on $K^{n}$, and $q(z)=l(z)-\int l(z) d m$ is a continuous, nonconstant, complex-valued function on $K^{n}$ such that $q(T z)=\lambda q(z)$.

We have $|\lambda|=1$ because $\sup _{z \in K^{n}}|q(T z)|=|\lambda| \sup _{z \in K^{n}}|q(z)|$. Since $q(z) \in L^{2}\left(K^{n}\right)$, $q(z)=\sum_{i} b_{i} \gamma_{i}(z)$ ( $L^{2}$-convergence), where $\gamma_{i} \in \hat{K}^{n}$ and $\sum_{i}\left|b_{i}\right|^{2}<\infty$. Hence for every natural number $p, \sum_{i} b_{i} \gamma_{i}\left(a+A(a)+\cdots+A^{p-1}(a)\right) \gamma_{i}\left(A^{p} z\right)=\lambda^{p} \sum_{i} b_{i} \gamma_{i}(z)$ ( $L^{2}$-convergence). If $\gamma_{i}, A \gamma_{i}, A^{2} \gamma_{i}, \ldots$ are all distinct then $b_{i}=0$, for otherwise the condition $\sum\left|b_{i}\right|^{2}<\infty$ is violated. Therefore $b_{i} \not \equiv 0$ implies $A^{p} \gamma_{i}=\gamma_{i}$ for some $p \geqq 1$ and for all such $p, \gamma_{i}\left(a+A(a)+\cdots+A^{p-1}(a)\right)=\lambda^{p}$. Since $q(z)$ is nonconstant there must be some nontrivial character with this property, and hence the necessity of the conditions is proved.

The fact that $g$ maps $K^{n}$ onto $K^{m}$ has not been used in the above reasoning.

We now prove the sufficiency of the conditions. Starting from the conditions and the given map $h$ of $K^{n}$ onto $K^{m}$ we shall construct a continuous map $F$ of $R^{n}$ onto $R^{m}$ which has a decomposition, $F(x)=L(x)+P(x)+c$, into linear, periodic and constant parts satisfying $P \not \equiv 0$ and

(4) $L A(x)=B L(x), x \in R^{n}$,

(5) $P(a+A(x))=P(a)+B P(x), x \in R^{n}$,

(6) $(B-I)(c)=L(a)+P(a)-b+\nu$ for some $\nu \in Z^{m}$.

Since we then have $F(a+A(x))=b+B F(x)+\nu, x \in R^{n}, F$ induces a nonaffine, continuous map $g$ of $K^{n}$ onto $K^{m}$ such that $g T=S g$.

We first construct the transformation $P$ which we want to be periodic, not identically zero, and to satisfy equation (5). Let $\gamma(z)$ denote the given element of $\hat{K}^{n}$ and $\lambda$ the given eigenvalue of the linear transformation $B$. The method is to construct from $\gamma(z)$ a nonconstant, continuous, complex-valued function $q(z)$ of $K^{n}$ such that $q(T z)=\lambda q(z)$ and then to produce a continuous, complex-valued function $f(x)$ of $R^{n}$ such that $f \not \equiv 0$ and $f(a+A(x))=f(a)+\lambda f(x)$. We then use the Jordan normal form of the linear transformation $B$ to produce the transformation $P$.

Suppose that $p \geqq 1$ is the smallest positive integer such that $A^{p} \gamma=\gamma$. Define $q: K^{n} \rightarrow C$ by

$$
\begin{aligned}
q(z)=\gamma(z)+\frac{\gamma(a)}{\lambda} \gamma(A z)+\frac{\gamma(a+A(a))}{\lambda^{2}} \gamma & \left(A^{2} z\right)+\cdots \\
& +\frac{\gamma\left(a+A(a)+\cdots+A^{p-2}(a)\right)}{\lambda^{p-1}} \gamma\left(A^{p-1} z\right) .
\end{aligned}
$$


$q$ is nonconstant, continuous and $q(T z)=\lambda q(z), z \in K^{n}$. If $l(z)=q(z)-q(a) / \lambda$ then $l \neq \equiv, l(0)=0$ and $l(T z)=l(a)+\lambda l(z), z \in K^{n} . l(z)$ determines a continuous function $f: R^{n} \rightarrow C$, defined by $f(x)=l\left(x+Z^{n}\right)$, such that $f \not \equiv 0, f(0)=0, f(x+\nu)=f(x)$, $x \in R^{n}, \nu \in Z^{n}$, and $f(a+A(x))=f(a)+\lambda f(x), x \in R^{n}$.

As before, let $w_{1}, \ldots, w_{m}$ denote the basis of $C^{m}$ for which the linear transformation $B$ has Jordan normal form $D_{B}=U^{-1} B U$. Suppose that $i_{0}$ is the largest integer such that the eigenvalue $\lambda$ corresponds to the basis element $w_{i_{0}}$. Since $f(x) \not \equiv 0$ either $\mathscr{R}\left[U\left(f(x) w_{i_{0}}\right)\right] \not \equiv 0$ or $\mathscr{I}\left[U\left(f(x) w_{i_{0}}\right)\right] \not \equiv 0$. In the former case define $P(x)=\mathscr{R}\left[U\left(f(x) w_{i_{0}}\right)\right], x \in R^{n}$, and otherwise define $P(x)=\mathscr{I}\left[U\left(f(x) w_{i_{0}}\right)\right], x \in R^{n}$. $P: R^{n} \rightarrow R^{m}$ is continuous; $P \not \equiv 0, P(0)=0$, and $P(x+\nu)=P(x), x \in R^{n}, \nu \in Z^{n}$. $P$ satisfies equation (5) because

$$
\begin{aligned}
U\left(f(a+A(x)) w_{i_{0}}\right) & =U\left(f(a) w_{i_{0}}\right)+U\left(\lambda f(x) w_{i_{0}}\right) \\
& =U\left(f(a) w_{i_{0}}\right)+B U\left(f(x) w_{i_{0}}\right) .
\end{aligned}
$$

We have now constructed the periodic part $P$ of $F$. It remains to construct the linear part $L$ and the constant part $c$. We may as well assume that the given map $h$ of $K^{n}$ onto $K^{m}$ is affine since, in the contrary case there is nothing to prove. Let $F_{h}(x)=L_{h}(x)+c_{h}, x \in R^{n}$, denote the lifting of $h$. Since $h T=S h$, equations (1) and (3) give $L_{h} A(x)=B L_{h}(x), x \in R^{n}$, and $(B-I)\left(c_{h}\right)=L_{h}(a)-b+\nu_{1}$ where $\nu_{1}$ is some element of $Z^{m}$. Put $L=L_{h}$. Equation (4) is then satisfied.

We shall now choose the constant part $c$ of $F$. Suppose, without loss of generality, that $P$ is defined by taking real parts. Put $c=c_{h}+\mathscr{R}\left[U\left(f(a) /(\lambda-1) w_{i_{0}}\right)\right]+\nu_{2}$, where $\nu_{2} \in Z^{m}$ is chosen so that $c=\left(c_{1}, \ldots, c_{m}\right) \in R^{m}$ satisfies $0 \leqq c_{i}<1, i=1, \ldots, m$. We shall show that this choice of $c$ satisfies equation (6).

$$
\begin{aligned}
(B-I)(c) & =(B-I)\left(c_{h}\right)+\mathscr{R}\left[(B-I) U\left(\frac{f(a)}{\lambda-1} w_{i_{0}}\right)\right]+(B-I)\left(\nu_{2}\right) \\
& =L_{h}(a)-b+\nu_{1}+\mathscr{R}\left[U\left(D_{B}-I\right)\left(\frac{f(a)}{\lambda-1} w_{i_{0}}\right)\right]+(B-I)\left(v_{2}\right) \\
& =L_{h}(a)-b+\mathscr{R}\left[U\left(\frac{(\lambda-1)}{(\lambda-1)} f(a) w_{i_{0}}\right)\right]+\nu_{1}+(B-I)\left(\nu_{2}\right) \\
& =L_{h}(a)-b+P(a)+\nu, \quad \text { where } \nu=\nu_{1}+(B-I)\left(\nu_{2}\right) \in Z^{m} .
\end{aligned}
$$

Hence $c$ satisfies equation (6).

It remains to show that $F=L+P+c$ maps $R^{n}$ onto $R^{m}$. This follows from Lemma 1 because $L_{h}$ maps $R^{n}$ onto $R^{m}$ and the range of $P$ is the image under $P$ of the closed unit $n$-cube. This completes the proof of the sufficiency of the conditions. We remark that $L_{h}(x)+\varepsilon P(x)+c_{\varepsilon}$ is, for every real $\varepsilon>0$ and a suitable constant $c_{\varepsilon}$, the lifting of a nonaffine continuous map $g_{\varepsilon}$ of $K^{n}$ onto $K^{m}$ such that $g_{\varepsilon} T=S g_{\varepsilon}$. In fact

$$
c_{\varepsilon}=c_{h}+\mathscr{R}\left[U\left(\frac{\varepsilon f(a)}{\lambda-1} w_{i_{0}}\right)\right]+\nu_{\varepsilon}
$$

will suffice for a suitable $\nu_{\varepsilon} \in Z^{m}$. 
We now consider the cases when $m=n$ and $T, S$ and $h$ are one-to-one transformations of $K^{n}$. The proof of the necessity of the conditions is as above. To prove sufficiency we have to construct a nonaffine homeomorphism $g$ of $K^{n}$ such that $g T=S g$. Since $L_{h}$ is invertible and $L_{h} Z^{n}=Z^{n}$ it will suffice to show that $L_{h}(x)+\varepsilon P(x)+c_{\varepsilon}$, where $P$ and $c_{\varepsilon}$ are constructed as above, is a one-to-one transformation for some real number $\varepsilon>0$. By Lemma 2 it will suffice to show that $P$ satisfies a Lipschitz condition of the form $\|P(x)-P(y)\| \leqq M\|x-y\|$ for all $x, y \in R^{n}$, where $M$ is some constant. But every character of $K^{n}$ is of the form $\gamma(z)$ $=\exp \left(2 \pi i \sum_{i=1}^{n} k_{i} z_{i}\right)$ where $z=\left(z_{1}, \ldots, z_{n}\right) \in K^{n}$ and $\left(k_{1}, \ldots, k_{n}\right) \in Z^{n}$, so that $f(x)$ is a finite linear combination of terms of the form $\cos 2 \pi\left(\sum_{i=1}^{n} k_{i} x_{i}\right)$ and $\sin 2 \pi\left(\sum_{i=1}^{n} p_{i} x_{i}\right)$ where $\left(k_{1}, \ldots, k_{n}\right) \in Z^{n}, \quad\left(p_{1}, \ldots, p_{n}\right) \in Z^{n}$ and $\left(x_{1}, \ldots, x_{n}\right) \in R^{n}$. Hence the coordinate functions of $P$ are real linear combinations of terms $\cos 2 \pi\left(\sum_{i=1}^{n} k_{i} x_{i}\right)$ and $\sin 2 \pi\left(\sum_{i=1}^{n} p_{i} x_{i}\right)$ and so have bounded first-order partial derivatives. Therefore, by the mean value theorem, $P$ satisfies a Lipschitz condition of the required form. This completes the proof of Theorem 2.

Corollary 1. Let T, $S$ and $h$ be as in Theorem 2, with the additional assumption that $T$ is ergodic. Then there exists a nonaffine continuous map $g$ of $K^{n}$ onto $K^{m}$ such that $g T=S g$ if and only if there exists $\gamma \in \hat{K}^{n}, \gamma \neq \equiv 1$, and an eigenvalue $\lambda$ of the lifted transformation $B$ such that $|\lambda|=1, \lambda^{p} \neq 1$ for all $p>0, A \gamma=\gamma$ and $\gamma(a)=\lambda$.

When $m=n$ and $S, T$, and $h$ are invertible transformations the above conditions are necessary and sufficient for the existence of a nonaffine homeomorphism $g$ of $K^{n}$ such that $g T=S g$.

Proof. We have to show that when $T$ is ergodic the necessary and sufficient conditions given in Theorem 2 reduce to those given in the corollary. The ergodicity of $T$ shows that $A^{p} \gamma=\gamma$ for some $p \geqq 1$ implies $A \gamma=\gamma$. From the proof of Theorem 2 we see that if $\lambda$ satisfies the condition given in Theorem 2 it is a proper value of the totally ergodic measure-preserving transformation $T$ (i.e. $q(T z)=\lambda q(z)$ for some $q(z) \in L^{2}\left(K^{n}\right)$ ) and so it cannot be a root of unity. Hence the corollary is proved.

We remark that when $m=1,2$ or 3 the lifted transformation $B$ cannot have an eigenvalue of modulus one which is not a root of unity. Therefore, for these values of $m$, all continuous maps of $K^{n}$ onto $K^{m}$, satisfying $g T=S g$, are affine if $T$ is ergodic.

Corollary 2. Let $T$ and $S$ be as in Corollary 1. If $T$ is strong-mixing then all continuous maps $g$ of $K^{n}$ onto $K^{m}$ such that $g T=S g$ are affine.

Proof. If $T=a+A$ is strong mixing then $A$ is ergodic. Hence $A \gamma=\gamma$ implies $\gamma \equiv 1$ and the result follows by Corollary 1 .

The following corollary extends the theorem of Adler and Palais, which was mentioned in $\S 0$, and also provides a converse of their theorem.

Corollary 3. Let $A$ and $B$ be endomorphisms of $K^{n}$ onto $K^{n}$ for which there exists 
a continuous map $h$ of $K^{n}$ onto $K^{n}$ satisfying $h A=B h$. Then there exists a nonaffine continuous map $f$ of $K^{n}$ onto $K^{n}$ such that $g A=B g$ if and only if the endomorphisms $A$ and $B$ are not ergodic. Also if $A, B$ and $h$ are invertible transformations there exists a nonaffine homeomorphism $g$ of $K^{n}$ such that $g A=B g$ if and only if the automorphisms $A$ and $B$ are not ergodic.

Proof. Suppose a nonaffine continuous map $g$ of $K^{n}$ satisfying $g A=B g$ exists. By Theorem 2 there exists $\gamma \in \hat{K}^{n}, \gamma \neq 1$, and an eigenvalue $\lambda$ of the lifted transformation $B$ such that $A^{p} \gamma=\gamma$ and $\lambda^{p}=1$ for some $p \geqq 1$. Therefore the endomorphisms $A$ and $B$ are not ergodic (see $\$ 2$ ).

Conversely suppose that the endomorphisms $A$ and $B$ are not ergodic. Again suppose that $h$ is affine, because in the contrary case there is nothing to prove. The linear part $L_{h}$ of the lifting of $h$ is an invertible linear transformation of $R^{n}$ such that $L_{h} A(x)=B L_{h}(x)$, for all $x \in R^{n}$. Therefore the linear transformations $A$ and $B$ have the same eigenvalues. Since the endomorphisms $A$ and $B$ are not ergodic there exists $\gamma \in \hat{K}^{n}, \gamma \neq \equiv 1$, and an eigenvalue $\lambda$ of the linear transformation $B$ such that $A^{p} \gamma=\gamma$ for some $p \geqq 1$ and $\lambda^{p}=1$ for all such $p$ (see $\$ 2$ ). The result now follows from Theorem 2. The above proof is also valid for the case when all the transformations are required to be invertible.

As pointed out in the paper [1] it would be interesting to know the answer to the following question. If $A$ and $B$ are ergodic automorphisms of $K^{n}$, is every invertible measure-preserving transformation $\phi$ of $K^{n}$, satisfying $\phi A=B \phi$, an affine transformation? A simple example shows that this question has a negative answer for noninvertible maps. In fact, the ergodic endomorphism $A x=-4 x(\bmod 1)$ of the circle group onto itself has a nonaffine, measure-preserving square root.

The next corollary strengthens, in the case of toroidal groups, the result of Hoare and Parry stated in $\S 0$.

COROLlaRY 4. If $T=a+A$ is an ergodic affine transformation of $K^{n}$ onto $K^{n}$ and $S=b+B$ is an ergodic affine transformation of $K^{m}$ onto $K^{m}$ having quasi-discrete spectrum, then all continuous maps $g$ of $K^{n}$ onto $K^{m}$ such that $g T=S g$ are affine transformations.

Proof. Since $S$ has quasi-discrete spectrum $\bigcap_{p=0}^{\infty}(B-I)^{p} K^{m}=\{0\}$ [5]. If $C_{p}$ is the annihilator subgroup of $(B-I)^{p} K^{m}$ then, since the sequence $\left\{C_{p}\right\}$ is an increasing sequence of subgroups of the finitely generated abelian group $\hat{K}^{m}$, we have $C_{p}=C_{p+1}$ for some $p$, i.e. $(B-I)^{p} K^{m}=(B-I)^{p+1} K^{m}$. Hence $(B-I)^{p} K^{m}=\{0\}$ i.e. $B-I$ is nilpotent. Hence all eigenvalues of the lifted transformation $B$ are equal to 1 [2]. The result then follows from Corollary 1 .

We end this section by giving an example of an ergodic affine transformation $T=a+A$ of $K^{5}$ which commutes with a nonaffine homeomorphism of $K^{5}$.

Let $T\left(\left(x_{1}, x_{2}, x_{3}, x_{4}, x_{5}\right)+Z^{5}\right)=\left(\alpha+x_{1},-x_{5}, x_{2}+8 x_{5}, x_{3}-6 x_{5}, x_{4}+8 x_{5}\right)+Z^{5}$ where the real number $\alpha$ will be determined below. The lifting $F_{T}$ of $T$ is given by

$$
F_{T}\left(x_{1}, x_{2}, x_{3}, x_{4}, x_{5}\right)=(\alpha, 0,0,0,0)+\left(x_{1},-x_{5}, x_{2}+8 x_{5}, x_{3}-6 x_{5}, x_{4}+8 x_{5}\right) \text {, }
$$


where

$$
x=\left(x_{1}, x_{2}, x_{3}, x_{4}, x_{5}\right) \in R^{5} .
$$

The eigenvalues of the linear part of $F_{T}$ are the eigenvalues of the matrix

$$
\left[\begin{array}{rrrrr}
1 & 0 & 0 & 0 & 0 \\
0 & 0 & 0 & 0 & -1 \\
0 & 1 & 0 & 0 & 8 \\
0 & 0 & 1 & 0 & -6 \\
0 & 0 & 0 & 1 & 8
\end{array}\right]
$$

There are three eigenvalues of unit modulus: $\lambda_{1}, \lambda_{2}=2-\sqrt{ } 3 \pm i(4 \sqrt{ } 3-6)^{1 / 2}, \lambda_{3}=1$. $\lambda_{1}$ and $\lambda_{2}$ are not roots of unity. To see this we note that the equation $\lambda^{4}-8 \lambda^{3}+6 \lambda^{2}$ $-8 \lambda+1=0$, which is irreducible over the rationals, has roots $\lambda_{1}, \lambda_{2}$ and two real roots not of unit modulus. Hence $\lambda^{4}-8 \lambda^{3}+6 \lambda^{2}-8 \lambda+1$ is not a factor of $\lambda^{n}-1$ for any $n$. Hence $\lambda_{1}=\exp \left(2 \pi i \theta_{1}\right)$ where $0<\theta_{1}<1$ and $\theta_{1}$ is irrational. Put $\alpha=\theta_{1}$ in the definition of $T . T$ is ergodic because $\left[a,(A-I) K^{5}\right]=K^{5}$ and the lifted transformation $A$ has no roots of unity as eigenvalues besides $\lambda=1$.

Let $\gamma$ be the element of $\hat{K}^{5}$ defined by $\gamma\left(\left(x_{1}, x_{2}, x_{3}, x_{4}, x_{5}\right)+Z^{5}\right)=\exp \left(2 \pi i x_{1}\right)$.

Then $A \gamma=\gamma$ and $\gamma(a)=\lambda_{1}$. Therefore, by Corollary 1, there is a nonaffine homeomorphism of $K^{5}$ which commutes with $T$.

5. Roots of affine transformations of tori. As pointed out in $\$ 4$ every endomorphism $A$ of $K^{n}$ onto $K^{n}$ is of the form

$$
A\left(\left(x_{1}, \ldots, x_{n}\right)+Z^{n}\right)=\left(\left(\sum_{j=1}^{n} a_{1 j} x_{j}, \sum_{j=1}^{n} a_{2 j} x_{j}, \ldots, \sum_{j=1}^{n} a_{n j} x_{j}\right)+Z^{n}\right)
$$

where $[A]=\left[a_{i j} \mid i, j=1, \ldots, n\right]$ is an invertible $n \times n$ matrix with integer entries.

THEOREM 3. The strong-mixing affine transformation $T=a+A$ of $K^{n}$ onto $K^{n}$ has a continuous pth root if and only if there exists an invertible $n \times n$ matrix with integer entries whose pth power is the matrix $[A]$. All continuous pth roots of $T$ are affine transformations.

Proof. $T$ strong-mixing $\Rightarrow A$ ergodic $\Rightarrow(A-I) K^{n}=K^{n}$. If $(A-I) b=a$ then the transformation $\phi(z)=b+z, z \in K^{n}$, is a homeomorphism of $K^{n}$ such that $\phi T=A \phi$. Therefore $T$ has a continuous $p$ th root if and only if $A$ has a continuous $p$ th root.

Suppose that $S$ is a continuous $p$ th root of $A$, i.e. $S^{p}=A$. Since $S A=A S$, Corollary 2 implies that $S(z)=d+D(z), z \in K^{n}$, where $d \in K^{n}$ and $D$ is an endomorphism of $K^{n}$ onto $K^{n}$. Hence $D^{p}(z)=A(z)$ and $[D]$ is the required matrix.

Conversely, if $[B]^{p}=[A]$, where $[B]$ is an invertible matrix with integer entries, then $B^{p}=A$ where $B$ is the endomorphism of $K^{n}$ corresponding to $[B]$.

The last assertion of Theorem 3 follows from Corollary 2 . 


\section{REFERENCES}

1. R. L. Adler and R. Palais, Homeomorphic conjugacy of automorphisms on the torus, Proc. Amer. Math. Soc. 16 (1965), 1222-1225.

2. F. J. Hahn, On affine transformations of compact abelian groups, Amer. J. Math. 85 (1963), 428-446.

3. P. R. Halmos, Lectures on ergodic theory, Chelsea, New York, 1956.

4. A. H. M. Hoare and W. Parry, Semi-groups of affine transformations, Quart. J. Math. Oxford 17 (1966), 106-111.

5. —, Affine transformations with quasi-discrete spectrum. I, J. London Math. Soc. 41 (1966), 88-96.

6. _ Affine transformations with quasi-discrete spectrum. II, J. London Math. Soc. 41 (1966), 529-530.

7. L. S. Pontrjagin, Topological groups, Princeton Univ. Press, Princeton, N. J., 1958.

\section{UNIVERSITY OF SUSSEX,}

Sussex, England 